\title{
Marfanoid syndrome, De Silva type
}

INSERM

\section{Source}

INSERM. (1999). Orphanet: an online rare disease and orphan drug data base. Marfanoid syndrome, De Silva type. ORPHA:2464

Marfanoid syndrome, da Silva type is characterized by the association of marfanoid habitus with visceral diverticula. It has been reported in four adults and two siblings from a consanguineous marriage in two different publications. Pediatric cases also presented with diaphrag matic hernia. Other connective tissue disorders with visceral diverticula have been reported previously, suggesting a relationship between these two conditions. 